\title{
The Controllable Synthesis of Silver Nanowires by Using the Polyol Method with Trace Agent
}

\author{
Hongli Guo ${ }^{1}$,Guangjian Xing ${ }^{1, *}$ and Zhixin Yang ${ }^{2}$ \\ ${ }_{1,2}$ School of Materical Science and Engineering,Beijing Institute of Petrochemical Technology,Beijing 102617,China \\ ${ }^{1, a}$ School of Materical Science and Engineering,Beijing Institute of Petrochemical Technology,Beijing 102617,China
}

\begin{abstract}
Ag nanowires were successfully synthesized by the polyol process with the addition of trace amounts of $\mathrm{HCl}$ and $\mathrm{HNO}_{3}$. In addition, the relationship between the final morphology of the silver products and the reaction parameters, such as the quality ratio of $\mathrm{HCl}_{\text {and }} \mathrm{HNO}_{3}$ were studied detailed. The results show that when the quality ratio of $\mathrm{HCl}$ to $\mathrm{HNO}_{3}$ is $1: 2$, almost all of the silver products were thin nanowires with uniform size 40 to $60 \mathrm{~nm}$ in diameter and $30 \mu \mathrm{m}$ in length.
\end{abstract}

\section{Introduction}

Transparent electrodes are critical for many electronic devices such as liquid crystal displays, touch screens, plasma displays, organic light emitting diodes, and solar cells $[1,2]$.Transparent electrodes with random networks of silver nanowires (AgNWs) exhibit high electrical conductivity and transparency, because silver has the lowest resistivity among metals. Ag nanowires (NWs) are mostly synthesized by a polyol reduction method with poly(vinylpyrrolidone) (PVP) as the capping agent[3]. Xia et al. reported the possibility of controlling the morphology of nanomaterials under the existence of PVP(capping agent)by controlling the reaction conditions, such as the molar ratio of the reactants, reaction temperature, reaction time, in ethylene glycol (EG)[4].

Mao and co-workers carefully studied Xia's selfseeding method and demonstrated that the selective adsorption of PVP on the side surfaces $(100)$ and end surfaces(111) plays a crucial role in the growth of $\mathrm{Ag}$ $\mathrm{NWs}$ [5]. In addition, various additives, such as $\mathrm{FeCl}_{3}[6]$, $\mathrm{CuCl}_{2}$ [7], $\mathrm{Na}_{2} \mathrm{~S}$ [8], $\mathrm{NaCl}$ [9], $\mathrm{PtCl}_{2}$ [10], $\mathrm{KNO}_{3}$ [11], $\mathrm{AgBr}$ [12], have been commonly employed and determined to play an essential role in the selective formation of $\mathrm{Ag}$ NWs. The cations or anions from these additives are crucial for the seed formation and uniaxial growth of multiple twinned particles for the polyol synthesis of $\mathrm{Ag}$ $\mathrm{NWs}[6,7]$.Without fine control of reactant concentrations, especially the additives, some by-products, such as nanocubes or nanospheres, can be produced from the solution. Recently, we have sought to expand on this work by looking at the effect of $\mathrm{Cl}$ - and $\mathrm{H}+$ on the polyol reduction of $\mathrm{AgNO}$. In this work, we synthesized $\mathrm{Ag}$ NWs with polyol method with trace amounts of $\mathrm{HCl}$ and $\mathrm{HNO} 3$ to controllably adjust the diameter and length of the Ag NWs. We found that the addition of both $\mathrm{HCl}$ and $\mathrm{HNO}_{3}$ facilitated the growth of $\mathrm{Ag}$ nanowires.

\section{Experimental Section}

\subsection{Material}

Poly(Nvinylpyrrolidone) (PVPK 30, Mw $\approx 40,000$, the concentration was calculated in terms of the repeating unit) was purchased from Shnopharm Chemical Reagent
Co. Ltd. Silver nitrate $\left(\mathrm{AgNO}_{3}, 99.9 \%\right)$, ethylene glycol (EG), diluted hydrochloric acid ( $\mathrm{HCl} 1.8 \% \mathrm{w})$ and diluted nitric acid $\left(\mathrm{HNO}_{3} 3.2 \% \mathrm{w}\right)$ were purchased from Sinopharm Chemical Reagent Co. Ltd. All chemicals were of analytical grade and were used without further purification.

\subsection{Method}

Silver nanowires were synthesized by reducing $\mathrm{AgNO}_{3}$ with EG in the presence of PVP. A $50 \mathrm{~mL}$ EG solution of $0.09 \mathrm{M} \mathrm{AgNO}_{3}$ was preheating at $120^{\circ} \mathrm{C}$ for $30 \mathrm{~min}$. Another $50 \mathrm{ml}$ EG solution of $0.54 \mathrm{M}$ PVP with trace amounts of $\mathrm{HCl}$ and $\mathrm{HNO}_{3}$ was injected drop by drop using a syringe into the preheated solution. The mixture solution was maintained at $142^{\circ} \mathrm{C}$ for $1 \mathrm{~h}$.

For these syntheses that produced a high-yield of $\mathrm{Ag}$ nanowires under protection of argon,the following color changse were obseved :initially clear and colreless to light yellow (within $2 \mathrm{~min}$ ), to orange-red (5 min later), begining to green (with $20 \mathrm{~min}$ ), and finally to opaque gray with wispiness indicating the formation of long nanowires nanowires (within about $1 \mathrm{~h}$ ). Upon nanowires formation, the reaction was naturally coolied to room temperature $\left(25^{\circ} \mathrm{C}\right)$. Silver nanowires were easily separated from EG by addition of a large amount of acetone and ethanol, followed by sonication and centrifugation. Then the Ag NWS products were dispersed in ethanol for further characterization.

\subsection{Characterization}

The morphology of the synthetic Ag NWs was analyzed by field emission scanning electron microscopy equipped with energy dispersive X-ray spectroscopy (EDX) (FESEM, S-4800, Hitachi, Japan) operated at an acceleration voltage of $5 \mathrm{kV}$. Powder X-ray diffraction (PXRD)patterns were recorded on an X-ray diffractometer (Shimadzu XRD-7000) using $\mathrm{Cu} \mathrm{Ka}$ radiation with a scan rate of $2 \theta=4 \% \mathrm{~min}$ and a range of $2 \theta=25 \sim 85^{\circ}$. 


\section{Results and Discussion}

The silver nanomaterials were obtained by reducing $\mathrm{AgNO}_{3}$ with EG in the presence of PVP. Besides, trace additives of $\mathrm{HCl}$ and $\mathrm{HNO}_{3}$ were added into the reaction system to investigate its influence on the morphology of the silver nanoproducts. In order to examine the presence of Ag nanowires, the typical XRD pattern of as-prepared products is shown in Fig.1. Four characteristic diffraction peaks are observed. The characteristic diffraction peaks positioned at $37.9,44.1,64.2,77.3$, and $81.53^{\circ}$ are ascribed to(111),(200),(220),(311)and (222)planes of face-centered cubic (fcc) silver (JCPDS, No.04-0783), respectively, which shows that the products were Ag.

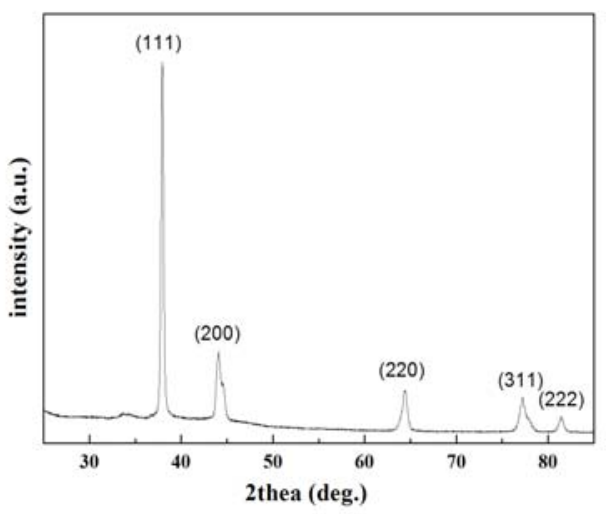

Fig 1. XRD pattern of AgNWs.

PVP is well-known to selectively passivate on $\{100\}$ planes of $\mathrm{Ag}$ seeds, leaving the unpassivated $\{111\}$ planes to grow longitudinally, but a little $\mathrm{HCl}$ and $\mathrm{HNO}_{3}$ also a play necessary role on uniform growth of silver. Fig 2 shows typical scanning electron microscopy(SEM) images of the as-obtained $\mathrm{Ag}$ nanocrystals based on different growth conditions. The most of samples without addition of $\mathrm{HCl}$ and $\mathrm{HNO}_{3}$ present a morphology of nanowire. but there is still a certain proportion of particles, while Ag NWs had shorter length $(2 \sim 5 \mu \mathrm{m})$ and larger diameter with about 95 120nm(as shown in Fig $2 \mathrm{a})$.With the addition of hydrochloric acid, the nanowire samples are distinctly observed (in Fig 2b), only little particles existed. However, when the quality ratio of $\mathrm{HCl}$ to $\mathrm{HNO}_{3}$ is $1: 2$, almost all of the silver products are thin nanowires (Fig 2c) with nearly uniform size, measured 35 to $50 \mathrm{~nm}$ in diameter and length up to $35 \mu \mathrm{m}$. Under the condition of only the presence of $\mathrm{HNO}_{3}$, a small amount of nanowires gradually disappear, instead of the larger particle products, and the length to diameter ratio of nanowires declined dramatically(Fig 2d). We attribute this difference to the absence of $\mathrm{Cl}^{-}$, which is needed to control the solution saturation when reagents are added rapidly by pipette.

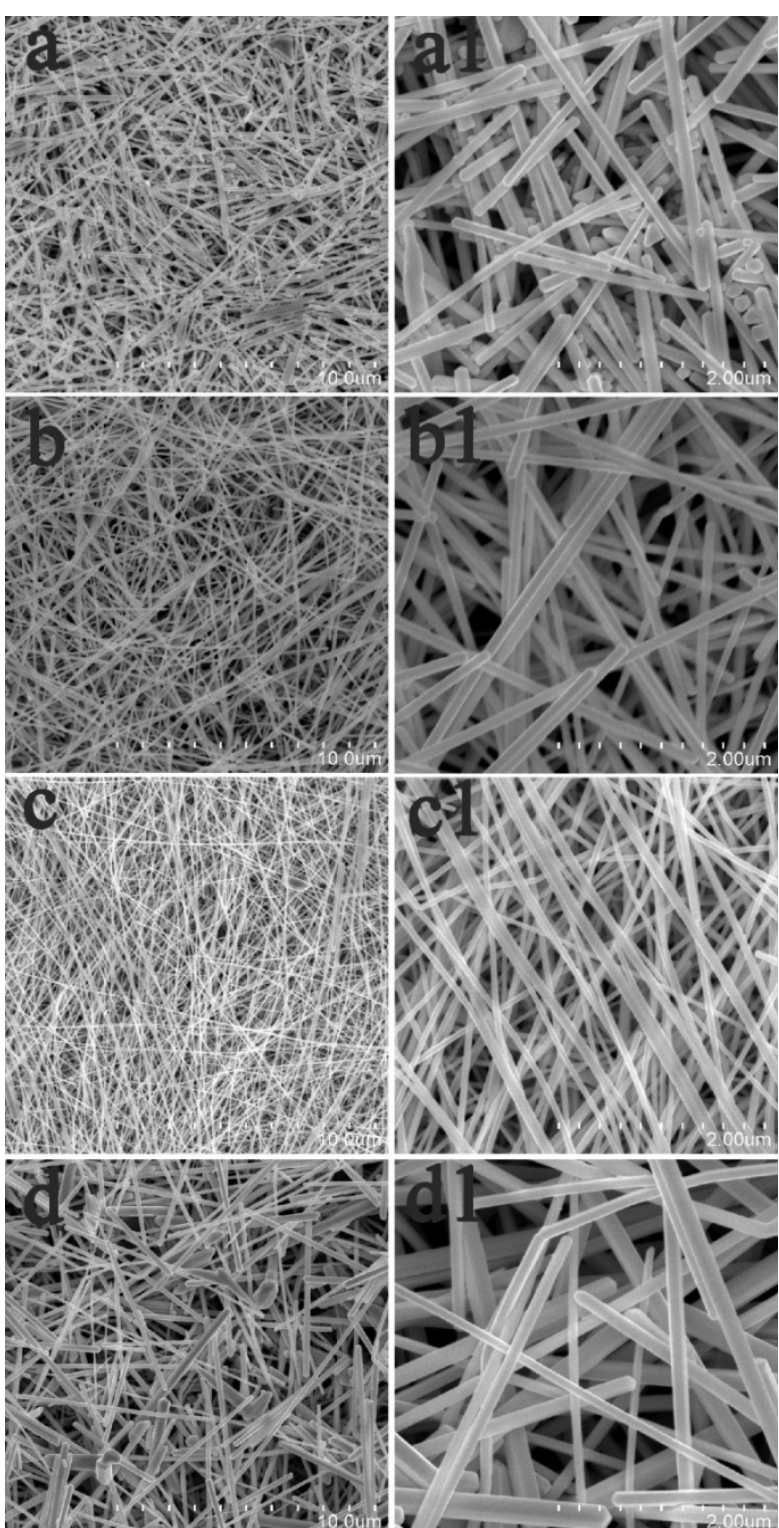

Fig 2. FESEM images with high magnification (a-d)and low magnification(a1-d1)of $\mathrm{Ag} \mathrm{NWs}$ synthesized at different quality ratios of $\mathrm{HCl}$ to $\mathrm{HNO}_{3}$ : a. $(0 \mathrm{gHCl}$, $\left.0 \mathrm{gHNO}_{3}\right)$; b. $\left(0.05 \mathrm{gHCl}_{0} 0 \mathrm{gHNO}_{3}\right)$; c. $(0.05 \mathrm{~g} \quad \mathrm{HCl}$, $\left.0.1 \mathrm{gHCO}_{3}\right)$; d. $\left(0 \mathrm{~g} \mathrm{HCl}, 0.1 \mathrm{gHNO}_{3}\right)$

Fig 3 shows the scattergrams of the diameter and length of $\mathrm{Ag} \mathrm{NWs}$ under the different trace additive agent of $\mathrm{HCl}$ and $\mathrm{HNO}_{3}$. We found that the addition of both $\mathrm{HCl}$ and $\mathrm{HNO}_{3}$ facilitated the growth of $\mathrm{Ag}$ nanowires, which not only affects the length and the diameter of the nanowires, but the uniformity of the AgNWs. When the mass ratio of $\mathrm{HCl}$ to $\mathrm{HNO}_{3}$ is $1: 2$, the ratio of length to diameter of $\mathrm{Ag}$ NWs is the largest. Consequently, at the same time of $\mathrm{Ag}$ nanocrystals grow preferentially along these facets due to PVP selected growth, trace control agent and reaction conditions, such as $\mathrm{pH}$, can also impact the morphology of silver nanoproducts. 


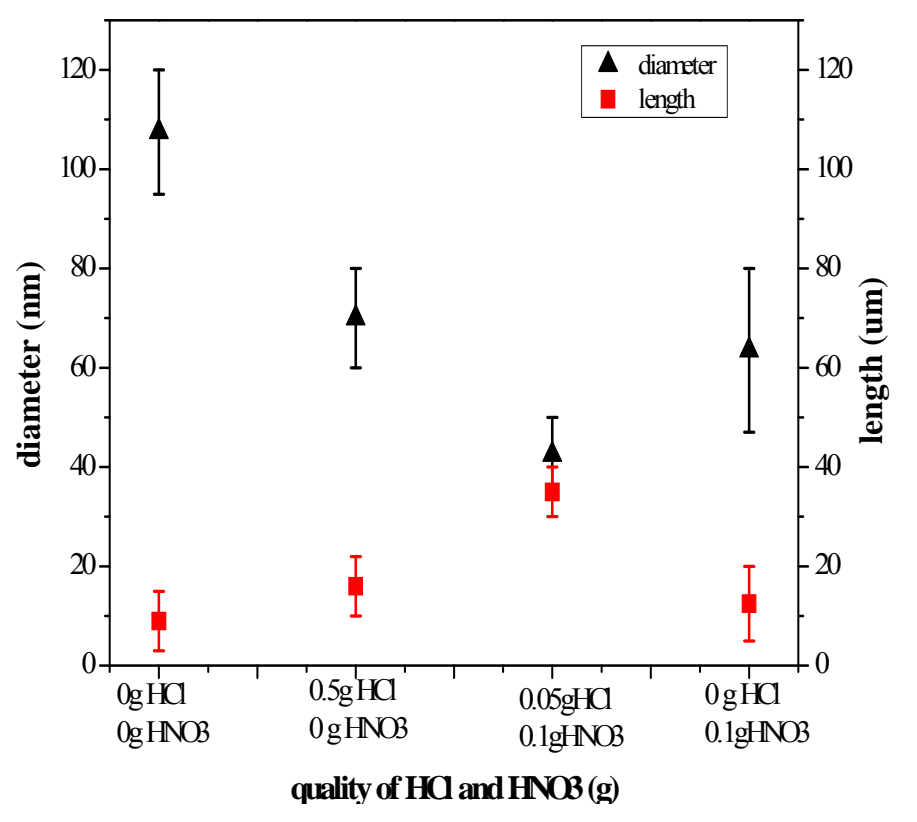

Fig 3. Distribution of diameters and lengthes for the batch of AgNWs shown in the SEM images of Fig 2.

At the initiation of the reaction without adding hydrochloric acid, because of the more free silver ion in the solution, the faster reaction rate lead to product as isotropic silver particles. Once hydrochloric acid added, $\mathrm{AgCl}$ precipitation formed immediately, which then reduced the nucleation rate of silver. As the reaction progresses, the reaction rate decreased, products began to anisotropic growth. This is mainly because:1.electrical activity of the oxidation of $\mathrm{AgCl} / \mathrm{Ag}$ is much lower than that of $\mathrm{Ag}^{+} / \mathrm{Ag}$, the formation of chelate $\mathrm{AgCl}$ increased the stability of silver ions; 2 . ethylene glycol is very weak reductant, and joined drop by drop, which in not enough into the reaction system; 3 . The concentration of silver ion in solution reduce a lot during the reaction process.

Fig 4 exhibits the growth process of silver nanowire. growth process of wires includes three aspects: (a). isotropic nucleation formation of silver nanoparticles; (b). anisotropic growth process on the surface of nanoparticles makes $\mathrm{Ag}$ crystals develop in length to diameter; (c).elemental silver reduced concentrate on the lower surface, eventually forming the nanowires.



Fig 4. The growth process of Ag NWs

\section{Conclusions}

Ag NWs were successfully synthesized by this selfseeding process through a polyol method. It is found that the addition of trace amounts of $\mathrm{HCl}$ and $\mathrm{HNO}_{3}$ greatly facilitates the formation of silver nanowires. Besides, the relationship between the final morphology of the products and the quality ratio of $\mathrm{HCl}$ to $\mathrm{HNO}_{3}$ was studied. $\mathrm{Cl}^{-}$combines $\mathrm{Ag}^{+}$to form amounts of $\mathrm{AgCl}$ colloids in the initial stage, resulting in decreasing free $\mathrm{Ag}^{+}$during initial formation of silver seeds and slowly releasing of $\mathrm{Ag}+$ to the solution in the subsequent reaction.The formation of chelate $\mathrm{AgCl}$ increased the stability of silver ions. The concentration of $\mathrm{H}^{+}$play an essential role in the selective formation of Ag nano products, mainly affected greatly the electrical activity of $\mathrm{AgCl} / \mathrm{Ag}$ to $\mathrm{Ag}^{+} / \mathrm{Ag}$, which then is effective in reducing the nucleation rate of silver. Finally, nanocrystal began to anisotropic grow to be AgNWs. In addition, silver nanowires with controllable sizes can be obtained by adjusting the concentration of $\mathrm{HCl}$ and $\mathrm{HNO}_{3}$.

\section{Acknowledgments}

The authors appreciate the financial support of the Importation and Development of High-Caliber Talents Project of Beijing Municipal Institutions (No. CIT \& TCD 201304102 \& 20134099).

\section{References}

[1] F.R. Fan, Y.Ding, D.Y.Liu, Z.Q. Tian, Z.L.Wang, Facet selective epitaxial growth of heterogeneous nanostructures of semiconductor and Metal: $\mathrm{ZnO}$ nanorods on Ag nanocrystals. J Am Chem Soc. 2009, 
131:12036-12037

[2] X.M.Feng, Y.F.Cheng, C.Ye, J.S. Ye, J.Y.Peng, J.Q. $\mathrm{Hu}$, Synthesis and Ag-content-depended electro chemical properties of $\mathrm{Ag} / \mathrm{ZnO}$ heterostructured nanomaterials. Mater Lett,.2012, 79:205-208

[3] Y. G. Sun, Y. D. Yin, B. T. Mayers, T. Herricks and Y. N. Xia. Chem.Mater., 2002, 14, 4736

[4] H. Mao, J. Feng, X. Ma, C. Wu and X. Zhao, J. Nanopart. Res.,2012, 14, 1-15

[5] Y.Sun, Y.Xia, Science. 2002, 298, 2176-2179

[6] B. Wiley, Y. Sun and Y. Xia, Langmuir, 2005, 21, 8077-8080

[7] K. E. Korte, S. E. Skrabalak and Y. Xia, J. Mater. Chem., 2008,18, 437-441
[8] D. Chen, X. Qiao, X. Qiu, J. Chen and R. Jiang, J. ColloidInterface Sci., 2010, 344,286-291

[9] W. M. Schuette and W. E. Buhro, ACS Nano, 2013, 7, 3844-3853

[10] Y. Sun, B. Gates, B. Mayers and Y. Xia, Nano Lett., 2002, 2,165-168

[11]C. L. Kuo and K. C. Hwang, Langmuir, 2012, 28, 3722- 3729

[12] S. Liu, J. Yue and A. Gedanken, Adv. Mater., 2001, $13,656-658$ 